\title{
10 Jahre Einsiedler Babyfenster - ein Rückblick
}

Stephan Rupp

Mit bestem Dank an die Schweizerische Hilfe für Mutter und Kind und die Vormundschaftsbehörde Einsiedeln für die Durchsicht des Manuskriptes.
Korrespondenz:

Dr. med. Stephan Rupp

Kinder- und Jugendmedizin

FMH

Spitalstrasse 30

CH-8840 Einsiedeln

stephan_rupp[at]bluewin.ch
Am 9. Mai 2001 wurde mit Hilfe der Schweizerischen Hilfe für Mutter und Kind (SHMK) und auf Betreiben des Gynäkologen Dr. W. Förster das Babyfenster in Einsiedeln eröffnet. Es bietet Müttern, die nicht in der Lage sind, ihr Kind zu behalten, die Möglichkeit, dieses ohne Aufgabe ihrer Anonymität in die Obhut des Spitals und schliesslich von Pflegeeltern zu geben. Ein Teil der Mütter gibt ihrem Kind ein Schreiben mit, in dem sie ihre Notlage aufzeigt, andere Frauen wollen ihre volle Anonymität wahren. Ein Kritikpunkt am Babyfenster ist, dass anders als bei einer Adoption, Kinder viel schwieriger und/oder gar nicht Kontakt zu ihren leiblichen Eltern aufbauen können, wenn zum Beispiel in der Pubertät die Fragen nach der Herkunft auftauchen. Letztlich geht es um das Problem, wo die Persönlichkeitsrechte der Mutter aufhören und die des Kindes beginnen. Es bleibt offen, was ohne Babyfenster mit diesen Kindern passiert wäre und ob die Hilfen genügen, die diesen Müttern von der Gesellschaft zur Verfügung gestellt werden.

Dieser Beitrag beschränkt sich auf den Zeitraum von der Abgabe der Kinder im Babyfenster bis zum Austritt aus dem Spital. In Absprache mit der Vormundschaftsbehörde in Einsiedeln, mit der eine sehr gute Zusammenarbeit besteht, werden keine Angaben zum weiteren Leben der abgegebenen Kinder gemacht. Im Allgemeinen kommen sie nach dem Spitalaufenthalt von meist 1 bis 2 Wochen vorüber- gehend in eine Empfangsfamilie, von wo aus sie später einer Familie zugeführt werden, die das Kind adoptieren kann. So ist das Risiko geringer, dass ein Kind einer adoptionswilligen Familie während der Zeit weggenommen werden muss, während der die leiblichen Eltern das Kind noch zurückfordern können.

Es bestanden und bestehen juristische Bedenken gegenüber dem Babyfenster. Diese sind sicher noch nicht endgültig ausgeräumt und werden spätestens dann wieder aufflammen, wenn ein Kind aus dem Babyfenster aus irgendeinem Grund stirbt oder dort tot hineingelegt wird. Auf jeden Fall ist die Zusammenarbeit mit der Polizei, die zwingend involviert werden muss, sehr positiv, und es hat sich in den letzten Jahren eine Routine entwickelt.

Es folgen einige Fakten zu den bisher sieben Babys aus dem Babyfenster. Im Schnitt wird also alle rund 1,5 Jahre ein Kind abgegeben. Interessanterweise waren es in den ersten 9 Betriebsjahren nur 4 Kinder, in den letzten zwei Jahren aber drei. Es ist möglich, dass dies mit der Akzeptanz und der Bekanntheit des Angebots zusammenhängt. Vielleicht besteht mehr Bereitschaft, eine Schwangerschaft versteckt auszutragen, wenn die Möglichkeit besteht, das Kind nach der Geburt anonym jemandem anzuvertrauen

\section{Abgabezeitpunkt}

Bemerkenswert ist, dass 5 von 7 Babys während des Tages bei Tageslicht abgegeben wurden, also nicht

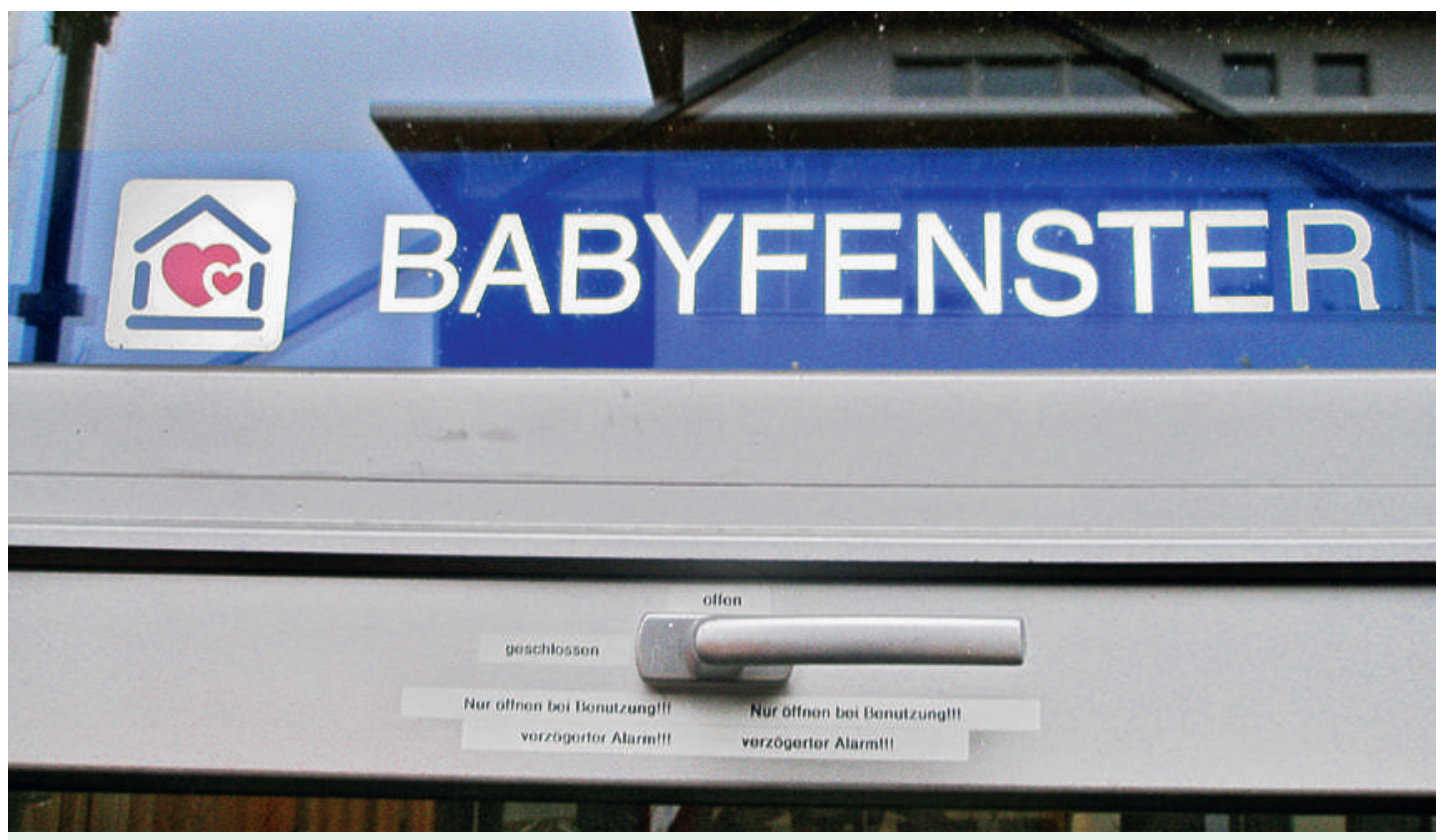

Sieben Neugeborene wurden in den ersten neun Betriebsjahren in das Einsiedler Babyfenster gelegt - drei davon in den letzten zwei Jahren. 
unauffälliger in der Nacht, obwohl das Fenster nicht sehr versteckt ist. Hier besteht bei unserer Einrichtung noch Optimierungspotential. Vor allem seit dem Neubau des Gesundheitszentrums ist die Abgabestelle relativ offen einsehbar. Vielleicht hängt der Abgabezeitpunkt auch mit der Erreichbarkeit des Spitals mit öffentlichen Verkehrsmitteln zusammen, nach Mitternacht sind An- und Abreise mit öffentlichen Verkehrsmitteln nicht mehr möglich.

\section{Alter bei der Abgabe}

Der genaue Geburtszeitpunkt ist ja nie bekannt. Bei drei Babys gehen wir davon aus, dass sie am ersten Lebenstag abgegeben wurden, was bedeutet, dass wohl schon vor der Geburt die Abgabe in die Klappe geplant war. Ein Kind wurde nach rund 3 Tagen, eines nach rund einer Woche und eines nach $12 \mathrm{Ta}$ gen (mit Angabe des Geburtstages) abgegeben. Hier bestand vielleicht noch Hoffnung auf die Möglichkeit, das Kind behalten zu können, oder die sofortige Trennung war zu schwierig. Ein Kind wurde erst im Alter von rund 6 Wochen abgegeben, dort wurde sicher versucht, Bedingungen zu schaffen, die es erlauben würden, das Kind zu behalten.

\section{Herkunft}

Natürlich ist es bei einem Neugeborenen schwierig, die Herkunft zu definieren. Lediglich ein Kind dürfte nicht europäischer Herkunft gewesen sein.

\section{Geburtsgewicht und Reife des Kindes}

Alle Kinder wurden, wo dies noch möglich war, auf-

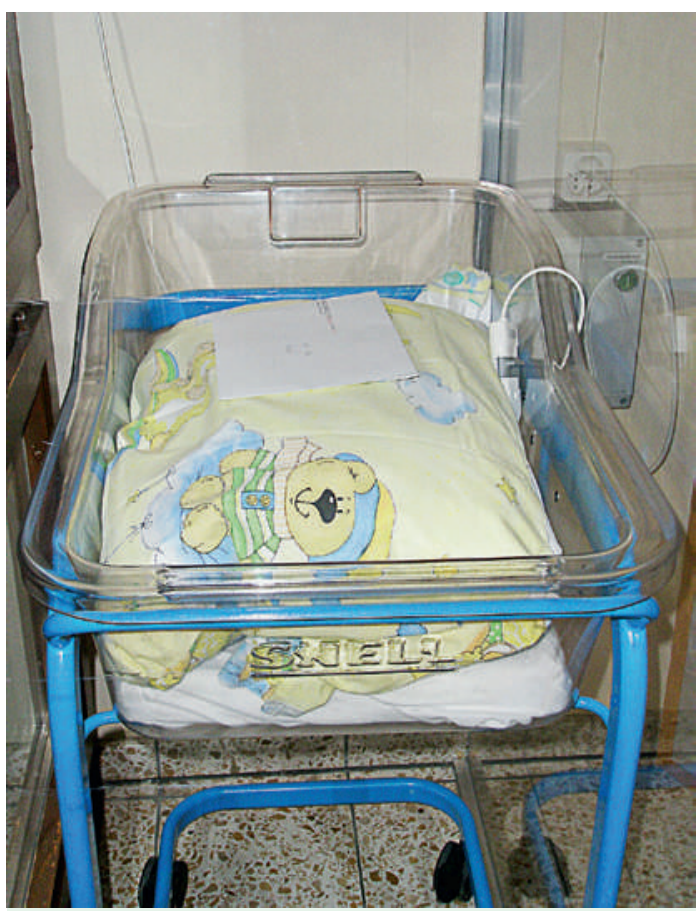

Das Kinderbett des Einsiedler Babyfensters mit dem Alarmsystem und dem Brief an die Eltern. grund des Dubowitzscores und sonst gemäss Schätzung als Termingeborene beurteilt. Drei der Kinder lagen im Bereiche einer Mangelgeburt, mit $1810 \mathrm{~g}$, $2250 \mathrm{~g}$ und $2470 \mathrm{~g}$, die anderen 4 wogen über $2500 \mathrm{~g}$. Der Anteil leichter Kinder ist, in diesem sehr kleinen Kollektiv, sicher übermässig hoch, was auf die nicht optimalen Verhältnisse während der Schwangerschaft zurückgeführt werden könnte. Glücklicherweise hatte kein Kind eine Hypoglykämie, was dafür spricht, dass die Kinder entweder sehr rasch gebracht wurden oder Nahrung erhalten haben.

\section{Abnabelung}

Wenn man weiss, wie sich die Mütter teils bei der Nabelpflege überwinden müssen, interessiert es, wie die Abnabelung erfolgt ist. Bei zwei Kindern war der Nabel bereits abgefallen, ein weiteres Kind hatte noch ein Nabelgranulom. Bei 4 Kindern war die Nabelschnur noch dran. Ein Nabel war abgerissen und vertrocknet, einer war abgetrennt, einmal wurde die Nabelschnur verknotet und abgeschnitten, ein Kind hatte an der Nabelschnur noch die Plazenta, die in einen Plastiksack eingewickelt war. Glücklicherweise kam es in keinem Fall zu einer Sepsis während der ersten Tage im Spital. Diese Zahlen zeigen, dass Öffentlichkeitsarbeit sinnvoll wäre, wie abgenabelt werden soll. Vielleicht müsste ein «Merkblatt» ins Netz gestellt werden.

\section{Drogen und Serologien}

Eigentlich war davon auszugehen, dass Kinder drogensüchtiger Mütter abgegeben werden würden. Bei den Kindern, die relativ rasch nach der Geburt abgegeben wurden, wurden Drogenscreenings durchgeführt, die allesamt negativ waren. Auch bei den übrigen Kindern wurde kein Entzugssyndrom festgestellt. Die Mehrheit der Kinder war sogar überraschend ruhig, vielleicht auch deshalb, weil sie früh gelernt hatten, nicht aufzufallen und zu stören.

Bei allen Kindern wurden Serologien durchgeführt. Die HIV-Serologie war in allen Fällen unauffällig. Zweimal fanden sich positive HBs-Antikörper bei sonst negativer Serologie, was, gemäss Rücksprache mit den Infektiologen, einem mütterlichen Impfschutz entspricht. Natürlich wurden die Kinder aktiv, und wo sinnvoll auch passiv, gegen Hepatitis geimpft.

\section{Zustand bei Eintritt}

Alle sieben Kinder waren vital. Hauptproblem waren die Körpertemperaturen bei Eintritt. Vier Kinder hatten Temperaturen um und unter 36 Grad (33,5 Grad; 35,9 Grad; 35,7 Grad; 36,0 Grad), wobei natürlich vor allem die jüngeren Neugeborenen dieses Problem hatten. Da die Kinder sofort nach Alarmauslösung an die Wärme kommen, ist von einem Transportproblem vor der Abgabe auszugehen. Hier müssen die Mütter unbedingt sensibilisiert werden. Als Folge der Hypothermie wurden vor allem Bradykardien beobachtet. Ein Kind musste mit tiefer Herz- 
frequenz und knappen Sättigungswerten ins Zentrumsspital verlegt werden. Es erholte sich aber rasch und vollständig.

\section{Fazit}

Es ist offensichtlich, dass das Einsiedler Babyfenster einem Bedürfnis entspricht. Es lässt sich nur darüber spekulieren, wann die Mütter den Entschluss gefasst haben, ihr Kind im Babyfenster abzugeben. Auf jeden Fall mussten sie sich früh entscheiden, ihre

\section{«Keine Mutter gibt ihr Kind freiwillig einfach weg, wie dies auch die teils mitgegebenen Briefe bezeugen.»}

Schwangerschaft versteckt auszutragen. $\mathrm{Zu}$ diesem frühen Zeitpunkt wäre wohl als Alternative nur eine Abtreibung in Frage gekommen, die die Mütter, aus welchen Gründen auch immer, vermeiden wollten. Nachdem der Entschluss gefallen ist, die Schwangerschaft im Geheimen auszutragen, gab es wohl teilweise noch die Hoffnung, dass sich die Situation bis zur Geburt klärt, dass vielleicht doch eine Möglichkeit besteht, das Kind zu behalten. Dies dürfte sicher bei dem Kind der Fall gewesen sein, das erst sechs Wochen nach der Geburt abgegeben wurde, vielleicht auch bei anderen. Es ist also sicher nicht davon auszugehen, dass das Angebot des Babyfensters zu niederschwellig ist; keine Mutter gibt ihr Kind freiwillig einfach weg, wie dies auch die teils mitgegebenen Briefe bezeugen. Es ist müssig, darüber zu spekulieren, ob durch das Fenster eine Kindstötung verhindert werden konnte, vielleicht wären die Kinder abgetrieben worden.

Einsiedeln ist zwar in der Agglomeration Zürich lokalisiert, doch nicht für alle Menschen einfach und in vernünftiger Zeit erreichbar. Die teils tiefen Körpertemperaturen der Kinder bei Eintritt ins Spital sind sicher auch eine Folge davon. In diesem Sinne wäre es wünschenswert, dass weitere Spitäler Babyfenster eröffnen, möglichst verteilt über das Land,

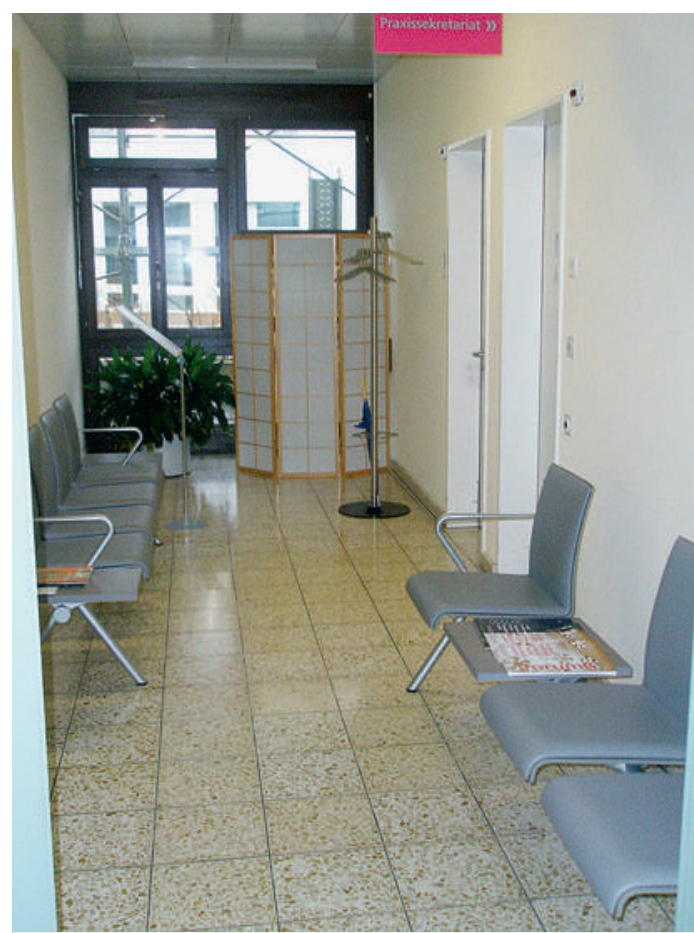

Hinter diesem Paravent verbirgt sich das Babyfenster.

möglichst auch in Zentren. Einsiedeln ist ein kleines Spital, dessen Möglichkeiten bei der Betreuung kranker Neugeborener sehr eingeschränkt sind. Es wäre deshalb günstig, dass Babyfenster an grösseren Zentren mit entsprechenden medizinischen Möglichkeiten und leichter Erreichbarkeit mit öffentlichen Verkehrsmitteln angeboten werden. Sicher gibt es noch juristische Fragen, die Sorgen bereiten. Was ist, wenn ein totes Kind abgegeben wird oder ein Kind nach der Ablage im Babyfenster stirbt?

Zum Schluss bleibt nur, allen Babyfensterkindern, die unser Einsiedler Team eine kurze Zeit auf ihrem Lebensweg begleiten durfte, alles Gute zu wünschen und Verständnis für die sicher schwere Situation zu haben, die ihre Mütter dazu gebracht hat, ihre Kinder jemand anderem anzuvertrauen. 